\title{
Clinical impact of the systemic immune-inflammation index in non-small cell lung cancer patients
}

\author{
Fumihiro Shoji \\ Department of Thoracic Surgery, Clinical Research Institute, National Hospital Organization, Kyushu Medical Center, Chuo-ku, Fukuoka, Japan \\ Correspondence to: Fumihiro Shoji, MD, PhD. Department of Thoracic Surgery, Clinical Research Institute, National Hospital Organization, Kyushu \\ Medical Center, 1-8-1 Jigyohama, Chuo-ku, Fukuoka 810-8563, Japan. Email: fshoji@surg2.med.kyushu-u.ac.jp. \\ Provenance and Peer Review: This article was commissioned and reviewed by the Academic Editor Dr. Shuangjiang Li (Department of Thoracic \\ Surgery and West China Medical Center, West China Hospital, Sichuan University, Chengdu, China). \\ Comment on: Berardi R, Santoni M, Rinaldi S, et al. Pre-treatment systemic immune-inflammation represents a prognostic factor in patients with \\ advanced non-small cell lung cancer. Ann Transl Med 2019;7:572.
}

Submitted Feb 19, 2020. Accepted for publication Mar 06, 2020.

doi: $10.21037 /$ atm.2020.03.180

View this article at: http://dx.doi.org/10.21037/atm.2020.03.180

Globally, lung cancer is the most common cause of cancerrelated death (1). The majority of all lung cancers are non-small cell lung cancer (NSCLC), and the outcome of NSCLC remains unfavorable compared with that of other solid organ malignancies. Many NSCLC cases are diagnosed as advanced or metastatic disease, although early detection or diagnosis methods for NSCLC have been developed. Only about $30 \%$ of NSCLC patients have a chance to receive surgery; however, the postoperative outcome of even early-stage NSCLC after curative surgical resection remains unsatisfactory. Although systemic therapy, including chemotherapy, molecular targeted therapy, and immunotherapy, has been extensively established, NSCLC outcomes remain poor. Therefore, further improvement of the NSCLC prognosis is required.

Recently, in addition to prognostic factors including clinico-pathological features of primary tumor, the inflammatory condition or immune-nutritional status of organ malignancies in patients has been highlighted as an important predictive factor of prognosis of patients because malignant cells grow by changing the host's inflammatory, immune, or metabolic status. The development of malignancies such as lung cancer, is intimately correlated with cancer-mediated inflammation and nutrition (2). Thus, inflammation has recently been noticed as a biomarker that could reflect the host immunological status. Inflammation has a significant function in the progression or development of several malignancies by promoting proliferation of malignant cells and survival, angiogenesis, and cancer metastasis (3).
Moreover, inflammation in the tumor microenvironment influences development of the tumor, and the generalized inflammatory status might demonstrate the cancer condition. Thus, inflammation is considered a hallmark of malignancy.

A complete blood cell count (CBC) is widely performed in systemic evaluation. Recently, CBC-derived inflammatory biomarkers, such as the neutrophil-lymphocyte ratio (NLR), platelet-lymphocyte ratio (PLR), and monocyte-lymphocyte ratio (MLR), have been utilized as prognostic biomarkers in diverse cancers $(4,5)$. These biomarkers are calculated by two factors related to neutrophils, lymphocytes, platelets, and monocytes.

Recently, the new CBC-derived biomarker systemic immune inflammation index (SII) has been developed. The SII level is based on three parameters-neutrophils, lymphocytes and platelets-and is calculated by multiplying the absolute platelet and neutrophil counts and dividing by the absolute lymphocyte count $(\mathrm{SII}=$ platelet count $\mathrm{x}$ neutrophil count/lymphocyte count). The prognostic value of SII was first reported by $\mathrm{Hu}$ et al. (6). Currently, SII has been explored as a novel prognostic factor in various malignancies originated from liver, kidney, prostate, esophagus, biliary tract, stomach and skin (7), while NLR, PLR, and MLR have been widely recognized as useful biomarkers in systemic inflammation and are related with poor prognosis of solid malignancies (4).

Considering the mechanism of this new biomarker, an elevated SII level may indicate three phenomena.

The first phenomenon is a simple relative decrease in 
lymphocytes. Lymphocytes provide anti-tumor immunity and function in cell-mediated immunity in various cancers. Lymphocytes also play crucial roles in the defense of malignant cells by inducing cytotoxic immune responses and preventing malignant cell proliferation, invasion, and migration $(3,8)$. Thus, lymphopenia might exhibit a decrease of $\mathrm{CD}^{+} \mathrm{T}$ helper lymphocytes, resulting in the diminishment of the immune response to cancer cells by reducing the influence of their essential role.

The second phenomenon is a relative increase in neutrophils. Neutrophils also play a critical role in the pathogenesis of several diseases, including cancer (9). Recent evidence shows that neutrophils may reinforce malignant cell invasion, proliferation, and metastasis, likewise help malignant cells avoid immune surveillance $(8,9)$. Moreover, neutrophils can promote the adhesion and seeding of distant organs by several growth factors and proteases are secreted (10). Thus, neutrophilia elevates the count of inflammatory factors including pro-angiogenic factors, growth factors such as cytokines or chemokines, and anti-apoptotic factors that establish a cancer microenvironment and accelerate cancer growth and progression (11).

The third phenomenon is a relative increase in thrombocytes. Thrombocytes also play an important role during tumor development and progression, and thrombocytosis is a paraneoplastic syndrome. Thrombocytes can interact with cancer cells and enhance cancer cell survival and metastasis through distinct mechanisms $(12,13)$. Thrombocytes can also protect circulating tumor cells (CTCs) from shear stresses during circulation, initiate the epithelial-mesenchymal transition and facilitate cancer cell extravasation to metastatic organs $(12,13)$. Hu et al. (6) clarified a significantly positive association between the SII and CTC levels, and the CTC level was significantly higher in the high SII level patients than in the low SII level patients $(\mathrm{P}=0.029)$.

These findings reveal that the SII level can represent lymphocyte, neutrophil, and thrombocyte conditions and may be strong parameters that reflect the host immune response condition. Thus, SII could influence the balance between the host inflammatory condition and immune response condition betterer than NLR or PLR, which reflects only two parameters.

\section{Preoperative SII levels in patients with operable- stage NSCLC}

An upgrade of the tumor stage indicates an advance of tumor malignant grade. From the above, the host inflammatory status influences the tumor condition. Therefore, the grade of the host inflammatory status increases as the tumor stage advances, a finding that was recently proven in some studies $(14,15)$. These studies revealed that the preoperative SII level is significantly associated with the TNM stage $[\mathrm{P}=0.002$ (Tomita et al.) and $\mathrm{P}=0.001$ (Guo et al.), respectively]. The actual preoperative SII level as a percentage in pathological stage II-III NSCLC was calculated as approximately $50-60 \%$ while that in pathological stage I was calculated as approximately $30-40 \%$. We have analyzed the clinical impact of the preoperative SII level in surgically resected patients with early-stage NSCLC (16). The present study revealed that a high preoperative SII level is significantly associated with postoperative recurrence $(\mathrm{P}=0.0458)$, and univariate analyses also showed that the SII status $(\mathrm{P}=0.0420)$ significantly affected recurrence-free survival (RFS). The relative risk for patients with a high preoperative SII level was $2.264 v s$. those with a low preoperative SII level [95\% confidential interval (CI): 1.028-5.976]. In addition, the high preoperative SII level group had a significantly shorter RFS than the preoperative low SII level group ( $\mathrm{P}=0.0438$, log-rank test), although our study did not show that the preoperative SII level was an independent prognostic factor in early-stage NSCLC patients. These results introduced by recent studies indicate that the high preoperative SII level is correlated with a more aggressive disease phenotype among operable NSCLC patients.

Thus, the preoperative SII level may be a useful and promising marker that can identify NSCLC patients with a high risk of postoperative recurrence and with a poor prognosis after surgical resection.

\section{Pretreatment SII level of patients with advanced/ metastatic lung cancer}

The pretreatment SII level is also highlighted not only as a predictive marker for treatment but also as a prognostic biomarker to predict outcome even in advanced or metastatic lung cancer. The prognostic impact of the pretreatment SII level for advanced NSCLC patients receiving first-line chemotherapy or targeted therapy has been reported by Berardi et al. (17). This study showed that the pretreatment SII level is significantly related to the tumor stage $(\mathrm{P}=0.0154)$, and multivariate analysis revealed that the pretreatment SII level is not only a predictor of a worse overall survival (OS) [hazard ratio (HR), 1.98; 95\% CI: $1.47-2.68 ; \mathrm{P}<0.001]$ but also a prognostic factor 
for a worse progression-free survival (PFS) (HR, 1.48; $\mathrm{P}=0.026)$. Tong et al. reported that the pretreatment high SII level is significantly correlated with a lower response rate of chemo-radiotherapy for locally advanced stage III NSCLC (18). This study revealed that the pretreatment SII level is significantly associated with the clinical stage (IIIA or IIIB) $(\mathrm{P}=0.019)$ and response to chemo-radiotherapy $(\mathrm{P}=0.018)$ and is significantly correlated with OS $(\mathrm{P}<0.001)$. Multivariate analysis showed that the pretreatment SII level is the most significant independent predictor of a worse OS (HR, 2.105; 95\% CI: 1.481-2.741; $\mathrm{P}<0.001)$. Deng et al. (19) also analyzed the association between the pretreatment SII level and advanced lung adenocarcinoma with mutant epidermal growth factor receptor (EGFR) treated with an EGFR-tyrosine kinase inhibitor. They showed that both the PFS and OS of patients with a pretreatment high SII level were shorter than those of patients with a pretreatment low SII level (PFS: $\mathrm{P}<0.001$; OS: $\mathrm{P}<0.001)$. Multivariate analysis revealed that the pretreatment SII level is an independent prognostic factor for both PFS (HR, 2.577; 95\% CI: 1.6773.958; $\mathrm{P}<0.001)$ and OS (HR, 2.802; 95\% CI: 1.659-4.733; $\mathrm{P}<0.001$ ). Liu et al. (20) examined the correlation between the pretreatment SII level and efficacy in NSCLC patients treated with an immune-checkpoint inhibitor. They found that the pretreatment SII level in NSCLC patients treated with nivolumab is independently correlated with a longer PFS (HR, 0.23; 95\% CI: 0.09-0.60; $\mathrm{P}=0.003$ ) and OS (HR, 0.13 ; $95 \%$ CI: $0.03-0.47 ; \mathrm{P}=0.002$ ) by multivariate analysis.

From these findings, the pretreatment SII level is also both a convenient prognostic factor and predictor to treat advanced or metastatic NSCLC.

\section{Future directions}

Some meta-analyses have been conducted to determine the prognostic value of the preoperative or pretreatment SII level in NSCLC patients $(21,22)$. However, the SII level is a relatively new marker and the sample size of studies concerning the SII level in NSCLC patients used for these meta-analyses is also relatively small. In addition, these studies are retrospective and validated by a limited number of countries such as China and Japan. Thus, in the future, a global and multi-centric, large-scale prospective trial is certified to assess the SII level as a predictive and prognostic biomarker for NSCLC patients according to the preoperative or pretreatment SII levels.
If the clinical significance of the SII level will be confirmed in the future, the preoperative or pretreatment SII level might apply as treatment strategies for NSCLC patients as follows. First, careful follow up or adjuvant therapy will be selected for postoperative NSCLC patients with a preoperative high SII level. Second, aggressive therapies, such as surgery, radiotherapy or systemic therapy, including chemotherapy, molecular targeted therapy, and immunotherapy will be avoided for NSCLC patients with a preoperative or pretreatment high SII level. Third, antiinflammatory treatment before or during aggressive therapy will be performed for NSCLC patients with a preoperative high SII level.

Systemic inflammation induces metabolic impairment and results in malnutrition. The immune-nutritional condition is also an independent prognostic factor in lung cancer patients (23). Therefore, the preoperative or pretreatment use of anti-inflammatory drugs and immune-nutritional support might improve the outcome of lung cancer patients. A previous study showed that non-steroidal anti-inflammatory drugs play a role in the prevention and treatment of cancer (24), and some clinical trials have been conducted to examine the advantages of preoperative immune-nutritional support mainly in patients with other organ malignancies. Presently, a prospective study on whether preoperative immune-nutritional support can improve the postoperative outcome in surgically resected NSCLC patients is under planning at our institution. Therefore, a prospective study is needed to analyze whether a preoperative or pretreatment anti-inflammatory or immune-nutritional program providing anti-inflammatory drugs and immune-nutritional support agents could improve the outcome in lung cancer patients with abnormal inflammatory biomarkers, including the SII level, or abnormal immune-nutritional parameters. These studies could contribute to the production of an anti-inflammatory or immune-nutritional support regimen that offers benefits for lung cancer patients with a high inflammatory status, such as a high SII level, or a low immune-nutritional condition.

\section{Acknowledgments}

We thank Nicole Okoh, PhD, from Edanz Group (https:// en-author-services.edanzgroup.com/) for editing a draft of this manuscript.

Funding: None. 


\section{Footnote}

Conflicts of Interest: The author has completed the ICMJE uniform disclosure form (available at http://dx.doi. org/10.21037/atm.2020.03.180). The author has no conflicts of interest to declare.

Ethical Statement: The author is accountable for all aspects of the work in ensuring that questions related to the accuracy or integrity of any part of the work are appropriately investigated and resolved.

Open Access Statement: This is an Open Access article distributed in accordance with the Creative Commons Attribution-NonCommercial-NoDerivs 4.0 International License (CC BY-NC-ND 4.0), which permits the noncommercial replication and distribution of the article with the strict proviso that no changes or edits are made and the original work is properly cited (including links to both the formal publication through the relevant DOI and the license). See: https://creativecommons.org/licenses/by-nc-nd/4.0/.

\section{References}

1. Siegel RL, Miller KD, Jemal A. Cancer statistics, 2019. CA Cancer J Clin 2019;69:7-34.

2. Alifano M, Mansuet-Lupo A, Lococo F, et al. Systemic inflammation, nutritional status and tumor immune microenvironment determine outcome of resected nonsmall cell lung cancer. PLoS One 2014;9:e106914.

3. Mantovani A, Allavena P, Sica A, et al. Cancer-related inflammation. Nature 2008;454:436-44.

4. Dolan RD, Lim J, McSorley ST, et al. The role of the systemic inflammatory response in predicting outcomes in patients with operable cancer: Systematic review and metaanalysis. Sci Rep 2017;7:16717.

5. Yuan C, Li N, Mao X, et al. Elevated pretreatment neutrophil/white blood cell ratio and monocyte/ lymphocyte ratio predict poor survival in patients with curatively resected non-small cell lung cancer: Results from a large cohort. Thorac Cancer 2017;8:350-8.

6. Hu B, Yang XR, Xu Y, et al. Systemic immuneinflammation index predicts prognosis of patients after curative resection for hepatocellular carcinoma. Clin Cancer Res 2014;20:6212-22.

7. Zhong JH, Huang DH, Chen ZY. Prognostic role of systemic immune-inflammation index in solid tumors: a systematic review and meta-analysis. Oncotarget 2017;8:75381-8.

8. Coussens LM, Werb Z. Inflammation and cancer. Nature 2002;420:860-7.

9. Mantovani A, Cassatella MA, Costantini C, et al. Neutrophils in the activation and regulation of innate and adaptive immunity. Nat Rev Immunol 2011;11:519-31.

10. Cools-Lartigue J, Spicer J, McDonald B, et al. Neutrophil extracellular traps sequester circulating tumor cells and promote metastasis. J Clin Invest 2013;123:3446-58.

11. Paramanathan A, Saxena A, Morris DL. A systematic review and meta- analysis on the impact of pre-operative neutrophil lymphocyte ratio on long term outcomes after curative intent resection of solid tumours. Surg Oncol 2014;23:31-9.

12. Labelle M, Begum S, Hynes RO. Direct signaling between platelets and cancer cells induces an epithelialmesenchymal-like transition and promotes metastasis. Cancer Cell 2011;20:576-90.

13. Schumacher D, Strilic B, Sivaraj KK, et al. Plateletderived nucleotides promote tumor-cell trans endothelial migration and metastasis via P2Y2 receptor. Cancer Cell 2013;24:130-7.

14. Tomita M, Ayabe T, Maeda R, et al. Systemic Immuneinflammation Index Predicts Survival of Patients After Curative Resection for Non-small Cell Lung Cancer. In Vivo 2018;32:663-7.

15. Guo W, Cai S, Zhang F, et al. Systemic immuneinflammation index (SII) is useful to predict survival outcomes in patients with surgically resected non-small cell lung cancer. Thorac Cancer 2019;10:761-8.

16. Shoji F, Kozuma Y, Toyokawa G, et al. Complete blood cell count-derived inflammatory biomarkers in early-stage non-small-cell lung cancer. Ann Thorac Cardiovasc Surg 2020. [Epub ahead of print].

17. Berardi R, Santoni M, Rinaldi S, et al. Pre-treatment systemic immune-inflammation represents a prognostic factor in patients with advanced non-small cell lung cancer. Ann Transl Med 2019; 7:572.

18. Tong YS, Tan J, Zhou XL, et al. Systemic immuneinflammation index predicting chemoradiation resistance and poor outcome in patients with stage III non-small cell lung cancer. J Transl Med 2017;15:221.

19. Deng C, Zhang N, Wang Y, et al. High systemic immuneinflammation index predicts poor prognosis in advanced lung adenocarcinoma patients treated with EGFR-TKIs. Medicine (Baltimore) 2019;98:e16875. 
20. Liu J, Li S, Zhang S, et al. Systemic immune-inflammation index, neutrophil-to-lymphocyte ratio, platelet-tolymphocyte ratio can predict clinical outcomes in patients with metastatic non-small-cell lung cancer treated with nivolumab. J Clin Lab Anal 2019;33:e22964.

21. Shoji F. Clinical impact of preoperative immunonutritional status in patients undergoing surgical resection of lung cancer. J Thorac Dis 2019;11:S408-12.

22. Zhang Y, Chen B, Wang L, et al. Systemic immuneinflammation index is a promising noninvasive marker to

Cite this article as: Shoji F. Clinical impact of the systemic immune-inflammation index in non-small cell lung cancer patients. Ann Transl Med 2020;8(11):668. doi: 10.21037/ atm.2020.03.180 predict survival of lung cancer: A meta-analysis. Medicine (Baltimore) 2019;98:e13788.

23. Wang Y, Li Y, Chen P, et al. Prognostic value of the pretreatment systemic immune-inflammation index (SII) in patients with non-small cell lung cancer: a meta-analysis. Ann Transl Med 2019;7:433.

24. Pereg D, Lishner M. Non-steroidal anti-inflammatory drugs for the prevention and treatment of cancer. J Intern Med 2005;258:115-23. 